\title{
Magnetic field effects on entropy generation in heat and mass transfer in porous cavity
}

\author{
Nawaf H. Saeid \\ Department of Mechanical, Materials and Manufacturing Engineering, The University of Nottingham Malaysia Campus, \\ 43500 Semenyih, Selangor, Malaysia.
}

Accepted 26 July, 2012

\begin{abstract}
The entropy generation for natural convection heat and mass transfer in a two dimensional porous cavity subjected to a magnetic field is selected for numerical investigation. The Darcy model is used in the mathematical formulation of the fluid flow in porous media. The mathematical model is derived in dimensionless form and the governing equations are solved using the finite volume method. The governing parameters arise in the mathematical model are the Rayleigh number, Lewis number, buoyancy ratio and Hartmann number. The results are presented as average Nusselt number $(\overline{N u})$, Sherwood numbers $(\overline{S h})$ and dimensionless form of local entropy generation rate $\left(N_{s}\right)$ for different values of the governing parameters. The numerical results show that increasing the magnetic field parameter (Hartmann number) leads to deterioration of the flow circulation strength in the cavity and this leads to a decrease in the rates of the heat and mass transfer as well as the rate of entropy generation. The results show a stagnate fluid everywhere in the cavity when the buoyancy forces generated due to temperature and concentration differences are in the same order and opposite directions. In this case, the values of $\overline{N u}, \overline{S h}$ and $N_{s}$ are the minimum. Increasing or decreasing the value of the buoyancy ratio parameter leads to enhance the fluid circulation and hence increase the values of $\overline{N u}, \overline{S h}$ and $\boldsymbol{N}_{s}$. The average Sherwood number can be increased with increasing Lewis number. It is observed that the strength of the fluid circulation in the cavity is reduced by increasing the Lewis number. This leads to the decrease in the average Nusselt number and the entropy generation by increasing Lewis number.
\end{abstract}

Key words: Natural convection, mass transfer, entropy generation, magnetic field, porous media.

\section{INTRODUCTION}

The natural convection in porous media has been studied and analysed widely in recent years. This interest was estimated due to many applications in, for example, packed sphere beds, high performance insulation for buildings, electronic packages, chemical catalytic reactors, to name a few. Representative studies in this area may be found in the books by Kaviany (1999), Nield and Bejan (2006) and Ingham and Pop (2001).
Double-diffusive convection in porous media concerns the processes of combined (simultaneous) heat and mass transfer which are driven by buoyancy forces. The buoyancy force is not only affected by the difference of temperature, but also it is affected by the difference of concentration in the fluid. A detailed review of doublediffusive natural convection in porous media can be found in research done by Mojtabi and Mojtabi (2005). Natural 
convection in a cavity saturated with porous media in the presence of magnetic field is relatively a new topic and needs more investigation. The effects of the magnetic field applied on electrically conducting fluids have been reported for various systems involving convection heat transfer. A detailed review of magnetic convection incrystal growth, including the effect of magnetic control can be found in the book by Ozoe (2005). There are many industrial applications of the electrically conducting fluids in the presence of a magnetic field such as crystal growth, electronic packages, metallurgical applications involving continuous casting and solidification of metal alloys and others. In such cases, the fluid experiences a Lorentz force, which tends to aid/oppose the fluid flow and hence increase/reduce the flow velocities. Oreper and Szekely (1983) showed that the strength of the magnetic field is one of the key factors in controlling the quality of the crystal. A numerical study is conducted by Rudraiah et al. (1995) to investigate the effect of magnetic field on the flow driven by the combined mechanism of buoyancy and thermocapillarity in a rectangular open cavity filled with a low Prandtl number fluid. The detailed flow structure and the associated heat transfer characteristics inside the cavity are presented. An investigation is conducted by Bian et al. (1996) to study the effect of an electromagnetic field on natural convection in inclined rectangular porous cavity saturated with an electrically conducting fluid. Bian et al. (1996) presented a linear stability analysis to determine the effect of the magnetic field on the onset of convection in a horizontal layer heated from below.

The irreversibility phenomena which are expressed by entropy generation are of important interest during the design of any thermodynamic system. Many studies concerning entropy generation in natural convection in porous media have been carried out, for example, Baytas (2000), Mahmud and Fraser (2004), Hooman et al. (2007), Famouri and Hooman (2008), Kaluri and Basak (2011), Mchirgui et al. (2012).

Baytas (2000) considered the entropy generation in natural convection in a tilted saturated porous cavity. The analysis of the study (Baytas, 2000) shows that, the calculations of local entropy generation maps are feasible and can be used for the selection of a suitable angle of inclination. The problem of entropy generation in a fluid saturated porous cavity for laminar magneto hydrodynamic natural convection heat transfer is analyzed by Mahmud and Fraser (2004). They studied the effect of Rayleigh and Hartmann numbers on average Nusselt number, entropy generation number, and Bejan number. Hooman et al. (2007) investigated analytically the entropy generation optimization of forced convection in porous-saturated ducts of rectangular cross-section. In this study, the authors indicated that it is possible to compare, evaluate, and optimize alternative rectangular duct design options in terms of heat transfer, pressure drop, and entropy generation. The entropy generation in natural convection by heated partition in a cavity, with adiabatic horizontal and isothermally cooled vertical walls is considered for numerical investigation by Famouri and Hooman (2008). Their results indicated that the fluid friction term has nearly no contribution to entropy production and the heat transfer irreversibility increases monotonically with the Nusselt number and the dimensionless temperature difference. A numerical study is carried out by Hooman et al. (2008) to investigate the entropy generation due to forced convection in a parallel plate channel filled by a saturated porous medium. The authors concluded that the dimensionless degree of irreversibility increases with increase in the porous media shape factor and the Brinkman number, and a decrease in the dimensionless heat flux or temperature difference. Kaluri and Basak (2011) considered the investigations on the entropy generation during natural convection in porous square cavities with distributed heat sources. Parametric study is carried out by Kaluri and Basak (2011) for four different configurations of discretely heated cavities based on the location of the heat sources on the walls of the cavities. Mchirgui et al. (2012) studied numerically the entropy generation in double-diffusive convection through a square porous cavity using DarcyBrinkman formulation. The cavity is saturated with a binary perfect gas mixture and subjected to horizontal thermal and concentration gradients. Their results indicated that the entropy generation considerably depends on the Darcy number and the porosity induces the increase of entropy generation, especially at higher values of Rayleigh number.

It is noted that, the entropy generation during the double diffusive convection in enclosed cavities submitted to a magnetic field has not received much attention. The aim of this paper is to study numerically the problem of entropy generation in heat and mass transfer in square porous cavity filled with electrically conducting fluid and subjected to a magnetic field. A schematic diagram of the porous cavity and coordinate system is shown in Figure 1. Horizontal temperature and concentration differences are specified between the vertical walls and zero mass and heat fluxes are imposed at the horizontal walls.

\section{Mathematical formulation}

The mathematical model in the present problem is formulated based on the following assumption:

1. The convective fluid and the porous media are in local thermal equilibrium,

2. The properties of the fluid and the porous media are constants,

3. The mass flux produced by temperature gradients (Soret effect) and the heat flux produced by a concentration gradients (Dufour effect) are neglected, 


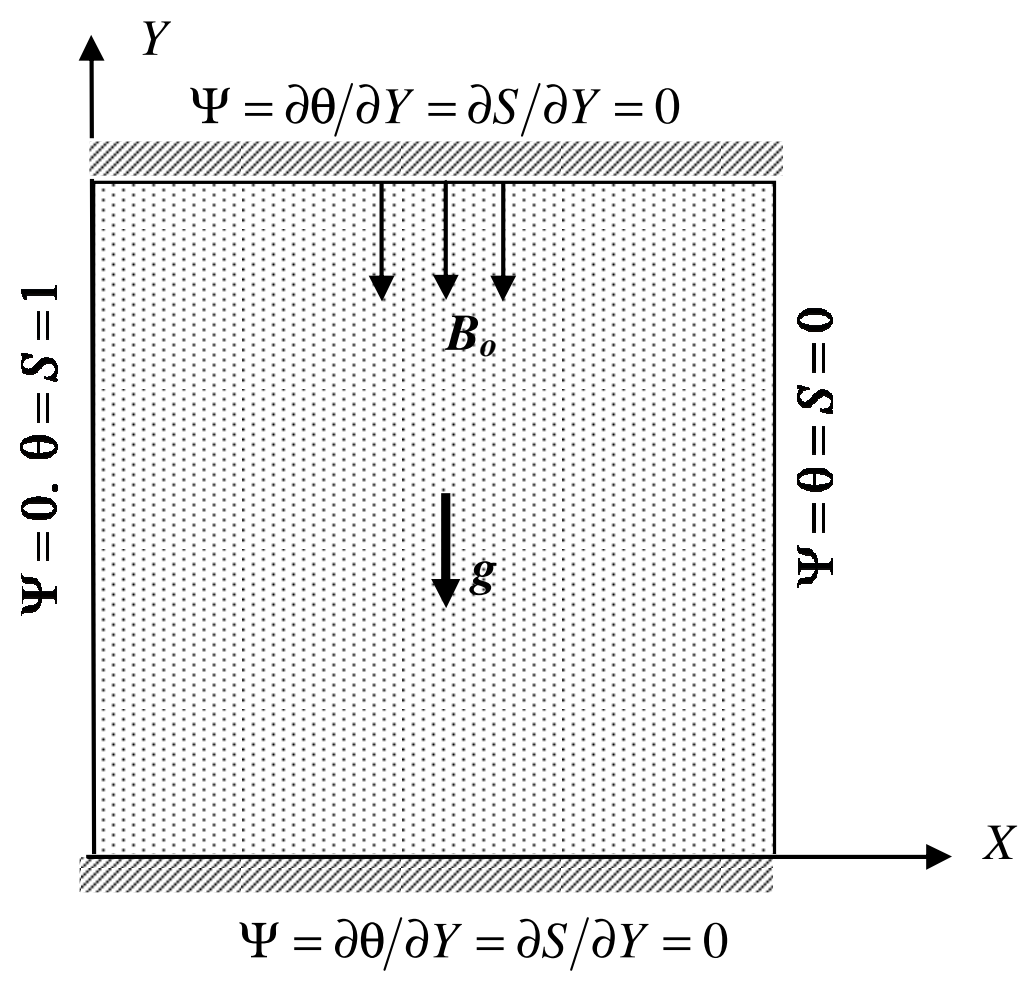

Figure 1. Schematic diagram of the physical model and coordinate system.

4. The viscous drag and inertia terms of the momentum equations are negligible, which are valid assumptions for low Darcy and particle Reynolds numbers,

5. The Darcy law is applicable under these assumptions, the conservation equations for steady flow can be written as:

$$
\begin{aligned}
& \nabla \cdot \overrightarrow{\mathbf{V}}=0 \\
& \overrightarrow{\mathbf{V}}=\frac{K}{\mu}(-\nabla p+\rho \overrightarrow{\mathbf{g}}+\overrightarrow{\mathbf{I}} \times \overrightarrow{\mathbf{B}}) \\
& \nabla \cdot \overrightarrow{\mathbf{I}}=0 ; \quad \overrightarrow{\mathbf{I}}=\sigma(-\nabla \varphi+\overrightarrow{\mathbf{V}} \times \overrightarrow{\mathbf{B}}) \\
& \nabla \cdot(\overrightarrow{\mathbf{V}} T-\alpha \nabla T)=0 \\
& \nabla \cdot(\overrightarrow{\mathbf{V}} C-D \nabla C)=0
\end{aligned}
$$

where $\overrightarrow{\mathbf{V}}\left(\mathrm{ms}^{-1}\right)$ is the velocity vector, $K\left(\mathrm{~m}^{2}\right)$ is the permeability of the porous medium, $\mu\left(\mathrm{kg} \cdot \mathrm{m}^{-1} \cdot \mathrm{s}^{-1}\right)$ is the dynamic viscosity, $p\left(\mathrm{Nm}^{-2}\right)$ is the pressure, ${ }^{\rho}\left(\mathrm{kg} \cdot \mathrm{m}^{-3}\right)$ is the density, $\overrightarrow{\mathbf{g}}\left(\mathrm{ms}^{-2}\right)$ is the acceleration vector, $\overrightarrow{\mathbf{B}}\left(\mathrm{Wbm}^{-2}\right.$ or Tesla) is the external magnetic field vector, $\overrightarrow{\mathbf{I}}(A)$ is the electric current vector, $\sigma\left(\Omega^{-1} \mathrm{~m}^{-1}\right)$ is the fluid electrical conductivity, $\varphi(\mathrm{V})$, is the electric potential, $T(\mathrm{~K})$ is the fluid temperature, $C\left(\mathrm{~mol} . \mathrm{m}^{-3}\right)$ is the concentration, $\alpha$ and $D\left(\mathrm{~m}^{2} \mathrm{~s}^{-1}\right)$ are diffusivity of heat and constituent through the fluid saturated porous matrix respectively. Garandet et al. (1992) proposed an analytical solution of the equations of magnetohydrodynamics that can be used to model the effect of a transverse magnetic field on buoyancy driven convection in a two-dimensional cavity. According to Garandet et al. (1992), Equation (3) can be reduced to $\nabla^{2} \varphi=0$. The unique solution is $\nabla \varphi=0$ since there is always an electrically insulating boundary around enclosure, where the gradients normal to the walls are zeros $(\partial \varphi / \partial n=0)$. It follows that the electric field vanishes everywhere as discussed by Alchaar et al. (1995).

The solution that saturates the porous matrix is modelled as a Boussinesq incompressible fluid whose density variation can be expressed using the OberbeckBoussinesq approximation:

$$
\rho \cong \rho_{o}\left\{1-\beta_{T}\left(T-T_{o}\right)-\beta_{c}\left(C-C_{o}\right)\right\}
$$

Where $\beta_{T}$ and $\beta_{c}$ are the thermal and concentration expansion coefficients. Subscript $o$ stands for a reference 
state. For two-dimensional flow the pressure $p$ in Equations (2) can be eliminated by cross differentiation and a single momentum equation can be derived. The governing equations may be written in dimensionless form using the following non-dimensional variables:

$$
\begin{aligned}
& X, Y=(x, y) / L ; \quad U, V=(u, v) L / \alpha ; \\
& \theta=\left(T-T_{c}\right) / \Delta T ; \quad S=\left(C-C_{c}\right) / \Delta C
\end{aligned}
$$

Where,

$\Delta T=\left(T_{h}-T_{c}\right)$ and $\Delta C=\left(C_{h}-C_{c}\right)$. The subscripts $h$ and $c$ stand for high and low values respectively. The dimensionless forms of the governing Equations (1) to (5) become:

$$
\begin{aligned}
& \frac{\partial^{2} \Psi}{\partial X^{2}}+\left(1-H a^{2}\right) \frac{\partial^{2} \Psi}{\partial Y^{2}}=-R a\left(\frac{\partial \theta}{\partial X}+N \frac{\partial S}{\partial X}\right) \\
& \frac{\partial \Psi}{\partial Y} \frac{\partial \theta}{\partial X}-\frac{\partial \Psi}{\partial X} \frac{\partial \theta}{\partial Y}=\frac{\partial^{2} \theta}{\partial X^{2}}+\frac{\partial^{2} \theta}{\partial Y^{2}} \\
& \frac{\partial \Psi}{\partial Y} \frac{\partial S}{\partial X}-\frac{\partial \Psi}{\partial X} \frac{\partial S}{\partial Y}=\frac{1}{L e}\left(\frac{\partial^{2} S}{\partial X^{2}}+\frac{\partial^{2} S}{\partial Y^{2}}\right)
\end{aligned}
$$

Where the stream function defined as $U=\partial \Psi / \partial Y$ and $V=-\partial \Psi / \partial X$, and the governing parameters are Rayleigh number, Buoyancy ratio, Lewis number and Hartmann number defined, respectively as:

$$
\begin{aligned}
& R a=\frac{\rho K g \beta_{T} \Delta T L}{\alpha \mu} ; N=\frac{\beta_{c}}{\beta_{T}} \frac{\Delta C}{\Delta T} ; L e=\frac{\alpha}{D} ; \\
& H a=B_{0} \sqrt{\sigma K / \mu}
\end{aligned}
$$

where $B_{0}$ is the magnitude of $\overrightarrow{\mathbf{B}}$. The governing equations in the present problem are subjected to the following boundary conditions:

at $X=0, \quad \Psi=0, \quad \theta=1, \quad S=1$

at $X=1, \quad \Psi=0, \quad \theta=0, \quad S=0$

at $Y=0, \quad \Psi=0, \quad \frac{\partial \theta}{\partial Y}=0, \quad \frac{\partial S}{\partial Y}=0$

at $Y=1, \quad \Psi=0, \quad \frac{\partial \theta}{\partial Y}=0, \quad \frac{\partial S}{\partial Y}=0$
The results is presented in terms of the average Nusselt number $\overline{\mathrm{Nu}}$ and the average Sherwood number $\overline{S h}$ on the vertical walls, which are defined as follows:

$$
\overline{N u}=\int_{0}^{1}\left(\frac{\partial \theta}{\partial X}\right)_{X=0,1} d Y ; \overline{S h}=\int_{0}^{1}\left(\frac{\partial S}{\partial X}\right)_{X=0,1} d Y
$$

\section{Entropy generation}

In the present convection heat and mass transfer problems, fluid friction, heat and mass transfer and the coupling between heat and mass transfer in addition to the magnetic field contribute to the rate of entropy generation. Accordingly, the volumetric entropy generation is the sum of the irreversibilities generation due to temperature gradients, viscous dissipation, magnetic field effect and concentration gradients. The irreversibility of the clear fluid is neglected based on the Darcy model and the viscous dissipation can be represented by velocity square term and the effective properties of the porous media (Baytas, 2000). Hence the volumetric entropy generation in heat and mass convection through a porous medium with the effect of magnetic field can be calculated by the following equation (Woods, 1975):

$\dot{s}_{g e n}=\frac{k}{T_{o}^{2}}(\nabla T)^{2}+\frac{\mu}{K T_{o}}\left(u^{2}+v^{2}\right)+\frac{\sigma B^{2}}{T_{o}} u^{2}+\frac{R D}{C_{o}}(\nabla C)^{2}+\frac{R D}{T_{o}}(\nabla T . \nabla C)$

Dimensionless form of Equation (14) can be obtained by utilising the dimensionless variable defined in (7) as:

$$
\begin{aligned}
\dot{S}_{G E N}=\left\{\left(\frac{\partial \theta}{\partial X}\right)^{2}+\left(\frac{\partial \theta}{\partial Y}\right)^{2}\right\} & +N_{\mu}\left\{\left(\frac{\partial \Psi}{\partial Y}\right)^{2}+\left(\frac{\partial \Psi}{\partial X}\right)^{2}+H a^{2}\left(\frac{\partial \Psi}{\partial Y}\right)^{2}\right\} \\
& +N_{C}\left\{\left(\frac{\partial S}{\partial X}\right)^{2}+\left(\frac{\partial S}{\partial Y}\right)^{2}\right\}+N_{T C}\left\{\left(\frac{\partial \theta}{\partial X}\right)\left(\frac{\partial S}{\partial X}\right)+\left(\frac{\partial \theta}{\partial Y}\right)\left(\frac{\partial S}{\partial Y}\right)\right\}
\end{aligned}
$$

where $N_{\mu}, N_{C}$ and $N_{T C}$ are irreversibility distribution ratios related to velocity gradients, concentration gradients and mixed product of concentration and thermal gradients, respectively. They are defined as follows:

$$
\begin{aligned}
& N_{\mu}=\frac{\mu T_{o}}{k}\left\{\frac{\alpha^{2}}{K(\Delta T)^{2}}\right\} ; N_{C}=\frac{R D T_{o}^{2}}{k C_{o}}\left(\frac{\Delta C}{\Delta T}\right)^{2} ; \\
& N_{T C}=\frac{R D T_{o}}{k}\left(\frac{\Delta C}{\Delta T}\right)
\end{aligned}
$$


Table 1. Comparison of $\overline{N u}$ with Mahmud and Fraser (2004) results ( $R a=250$ and $N=0)$.

\begin{tabular}{lccccc}
\hline $\mathbf{H a}$ & $\mathbf{0}$ & $\mathbf{2}$ & $\mathbf{4}$ & $\mathbf{6}$ & $\mathbf{1 0}$ \\
\hline Present results & 5.883 & 3.195 & 1.516 & 1.140 & 1.021 \\
Mahmud and Fraser (2004) & 5.90 & 3.15 & 1.50 & 1.15 & 1.05 \\
\hline
\end{tabular}

The local volumetric entropy generation would be integrated over the whole cavity to obtain the entropy generation number for the whole cavity volume as:

$N_{S}=\int_{0}^{1} \int_{0}^{1} \dot{S}_{G E N} d X d Y$

\section{NUMERICAL METHOD}

The dimensionless governing Equations (8) to (10) subjected to the boundary conditions (12) are integrated numerically using the finite volume method (Patankar, 1980; Versteeg and Malalasekera, 2007). The power law scheme proposed by Patankar (1980) is used for the convection terms formulation of the energy and mass transfer equations. The resulting algebraic equations were solved by line-by-line using the Tri-Diagonal Matrix Algorithm iteration. The iteration process is terminated under the following condition:

$$
\sum_{i, j}\left|\phi_{i, j}^{n}-\phi_{i, j}^{n-1}\right| / \sum_{i, j}\left|\phi_{i, j}^{n}\right| \leq 10^{-7}
$$

where $\phi$ is the general dependent variable which can stands for either $\theta, S$ or $\Psi$ and $n$ denotes the iteration step. The developed code is an extension of the code verified and validated in previous studies (Saeid and Mohamad, 2005; Saeid and Pop, 2004). The local volumetric entropy generation is calculated after calculating the temperature, concentration and stream function in each control volume in the mesh. Finally the entropy generation number for the cavity volume is calculated using Equation (17) via the numerical integration.

The present numerical results are compared with the results obtained by Mahmud and Fraser (2004) for the effect of magnetic field on the convective heat transfer in porous cavity. The results presented in Table 1 reflect the accuracy of the present results using uniform mesh of $40 \times 40$ square control volumes.

\section{RESULTS AND DISCUSSION}

The effect of the magnetic field on the entropy generation is represented by the second bracket in Equation (15). The irreversibility distribution ratios related to velocity gradients $\left(N_{\mu}\right)$ will be considered as a governing parameter in the range $0.001 \leq N_{\mu} \leq 1$. In order to reduce the governing parameters, the effect of the irreversibility distribution ratios related to concentration gradients $\left(N_{C}\right)$ and mixed product of concentration and thermal gradients $\left(N_{T C}\right)$ are fixed. The values of irreversibility coefficients $N_{C}=0.5$ and $N_{T C}=0.01$ are considered as recommended by Mchirgui et al. (2012) for the case of a square porous cavity filled with a binary perfect gas mixture without the effects of the magnetic field.

The results are generated to show the effect of the following governing parameters $0 \leq H a \leq 10$, $-5 \leq N \leq 5, \quad 0.001 \leq N_{\mu} \leq 1, \quad 1 \leq R a \leq 1000 \quad$ and $0.1 \leq L e \leq 10$ on the average Nusselt number, the average Sherwood number and the entropy generation number.

It is important to note that the dimensionless temperature and dimensionless concentration distributions are identical for the case of $L e=1$, which leads to $\overline{N u}=\overline{S h}$.

The classical natural convection in porous cavity without mass transfer and zero magnetic field is considered first as a reference case. The variation of the average Nusselt number $(\overline{\mathrm{Nu}})$ and the local entropy generation number $\left(N_{s}\right)$ with Rayleigh number $(R a)$ are shown in Figure 2 with fixed values of $N=0, L e=1$ and $H a=0$. Figure 2a shows the classical variation of $\overline{N u}$ with $\mathrm{Ra}$. It is important to note that the irreversibility distribution ratio $\left(N_{\mu}\right)$ and the Rayleigh number $(\mathrm{Ra})$ are related to each other as they are functions of the effective properties of the porous media. Hence the dependence of the heat transfer on the properties of the fluid and the porous media is justified through the dependence of $\overline{\mathrm{Nu}}$ on Ra. Mathematically; the effect of the effective properties on the thermal field can be represented by $\mathrm{Ra}, \mathrm{N}, \mathrm{Le}$ and $\mathrm{Ha}$ as can be seen in the dimensionless governing Equations (8) to (10). The irreversibility distribution ratio $\left(N_{\mu}\right)$ is not appeared in these equations and, therefore, it affects the thermal field implicitly through the effective properties of the porous media.

The effect of the irreversibility distribution ratio $\left(N_{\mu}\right)$ on the entropy generation number $\left(N_{s}\right)$ variation with Rayleigh number $(R a)$ is shown in Figure $2 \mathrm{~b}$. The results show that increasing $N_{\mu}$ leads to higher entropy generation in the cavity. From the definition of $N_{\mu}$, in 


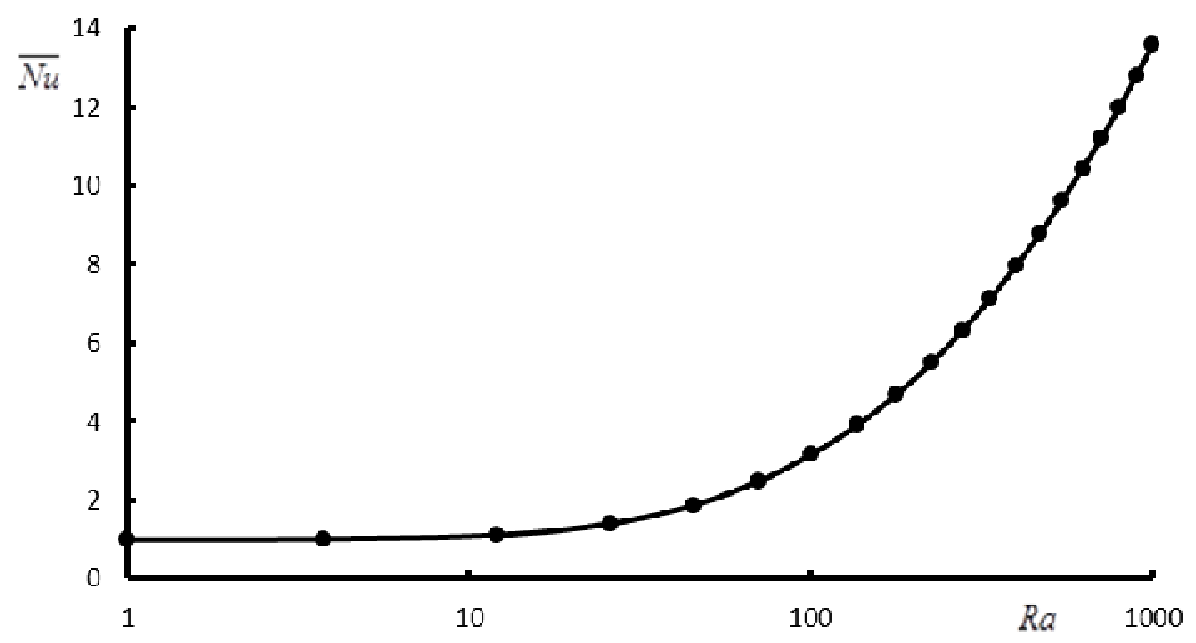

(a)

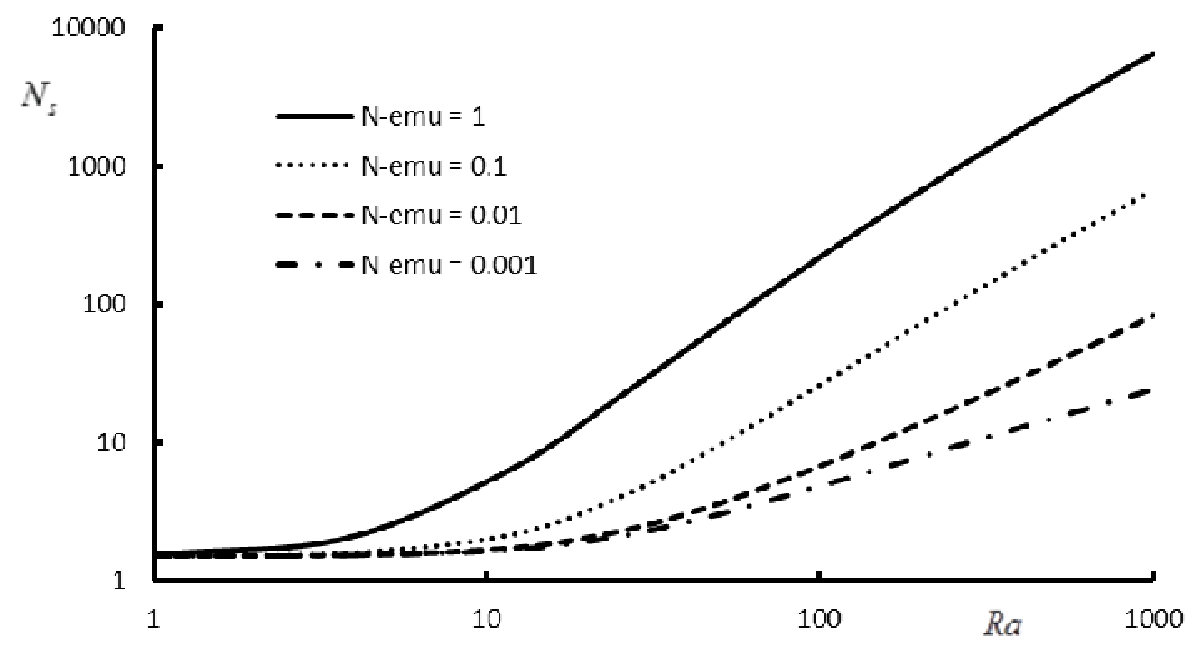

(b)

Figure 2. Variation of $\overline{N u}$ and $N_{s}$ with $R a$ at constant $N=0, H a=0$ and $L e=1$.

order to reduce the rate of entropy generation, it is necessary to reduce the reference temperature and reduce the viscosity and the thermal diffusivity of the fluid. The rate of entropy generation can be minimized for the flow through a high permeable porous medium.

The variation $\overline{\mathrm{Nu}}$ and $N_{s}$ with buoyancy ratio $(N)$ are shown in Figure 3 with fixed values of $N_{\mu}=0.1, L e=1$ and $\mathrm{Ha}=0$. The results depicted in Figure 3 show minimum values of $\overline{N u}$ and $N_{s}$ are observed at $N=-1$ for all the values of $\mathrm{Ra}$. Negative values of $N$ mean that the buoyancy forces generated due to temperature and concentration differences are in opposite directions. For $L e=1, H a=0$ and $N=-1$, Equation (8) reduced to $\nabla^{2} \Psi=0$ with $\Psi=0$ at the importable walls, leads to a stagnate fluid with $\Psi=0$ everywhere in the cavity. In this case, the heat and mass transfer are pure diffusion processes, in which the values of $\overline{N u}$ and $N_{s}$ are the minimum. Increasing or decreasing the value of the buoyancy ratio parameter $(N)$ leads to enhance the fluid circulation due to the increase of the resultant buoyancy force in the cavity. This leads to the increase the values of $\overline{N u}$ and $N_{s}$ as shown in Figure 3 .

The effect of the magnetic field on the variation of $\overline{N u}$ and $N_{s}$ is show in Figure 4 for different values of $R a$ and fixed values of $N_{\mu}=0.1$, Le $=1$ and $N=1$. Maximum rates of heat transfer as well as entropy generation are observed at $\mathrm{Ha}=0$. Increasing the 


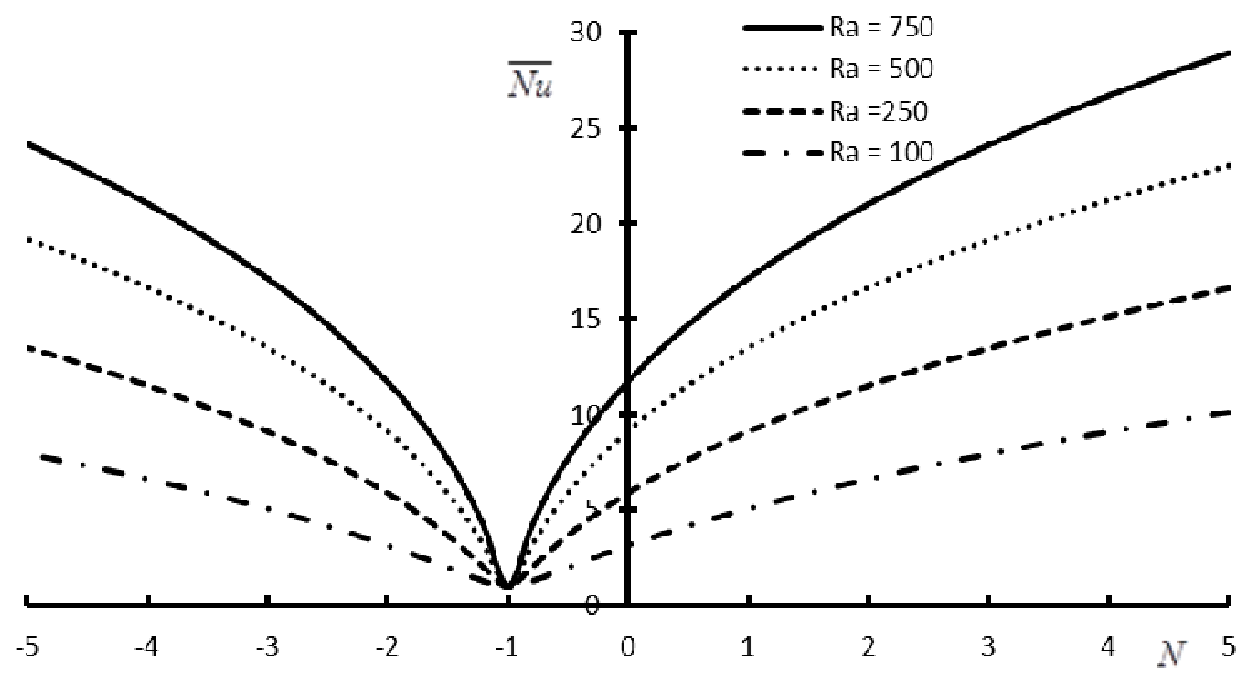

(a)

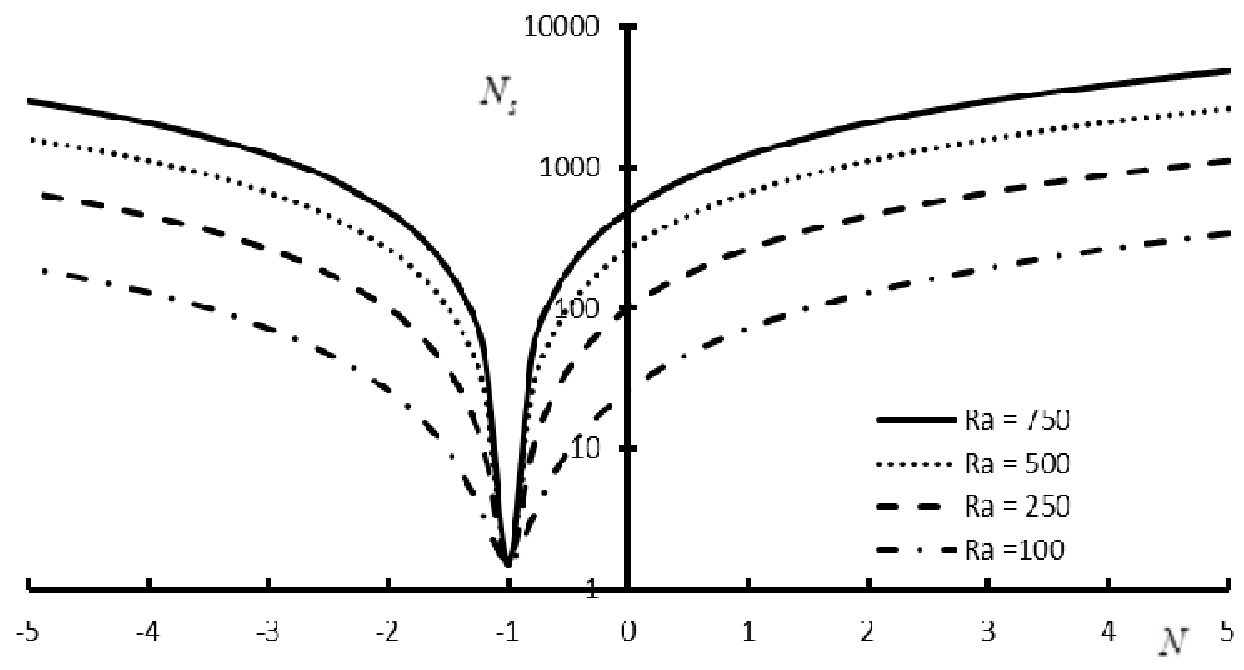

(b)

Figure 3. Variation of $\overline{N u}$ and $N_{s}$ with $N$ at constant $N_{\mu}=0.1, H a=0$ and $L e=1$.

magnetic field leads to increase the Lorentz forces and slowdown the fluid flow which leads to decrease $\overline{\mathrm{Nu}}$ rapidly as shown in Figure $5 \mathrm{a}$. For high values of $\mathrm{Ha} \mathrm{Ha}$ $>10$ ), the effect of Ra on the heat and mass transfer process is vanished and the values of $\overline{\mathrm{Nu}}$ approaching unity. The heat and mass transfer processes are associated with the entropy generation and again the rate of entropy generation is maximum when the rate of heat and mass transfer is maximum as shown in Figure 4b.

Finally, the effect of Lewis number $(L e)$ of the entropy generation for natural convection heat and mass transfer is studied for different values of Hartmann number $(\mathrm{Ha})$. The results presented in Figure 5 show the variations of $\overline{N u}, \overline{S h}$ and $N_{s}$ with $L e$ at constant $N_{\mu}=0.1, N=1$ and $R a=100$. At low values of $L e(0<L e<1)$, the values of $\overline{N u}$ are almost constant at fixed values of other parameters while $\overline{S h}$ is increasing slightly with the increase of $L e$ as shown in Figures 5 (a) and (b). In this range of $L e$, the heat and mass transfer processes are 


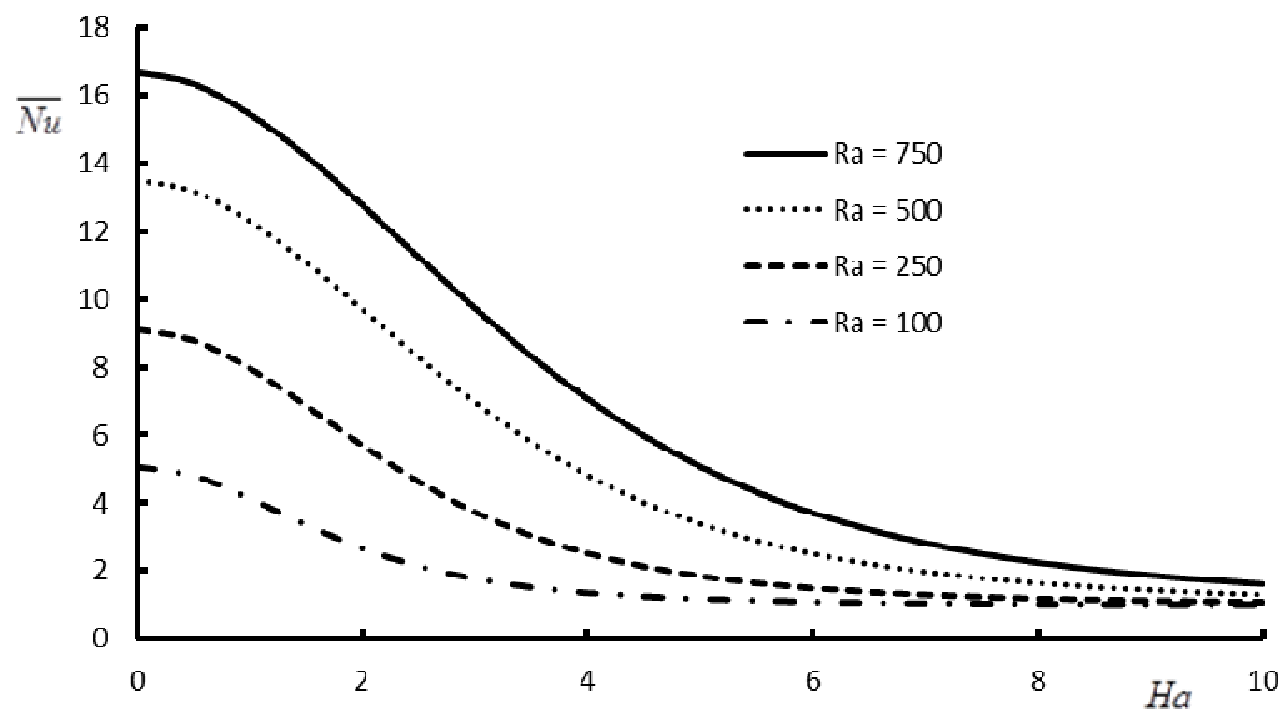

(a)

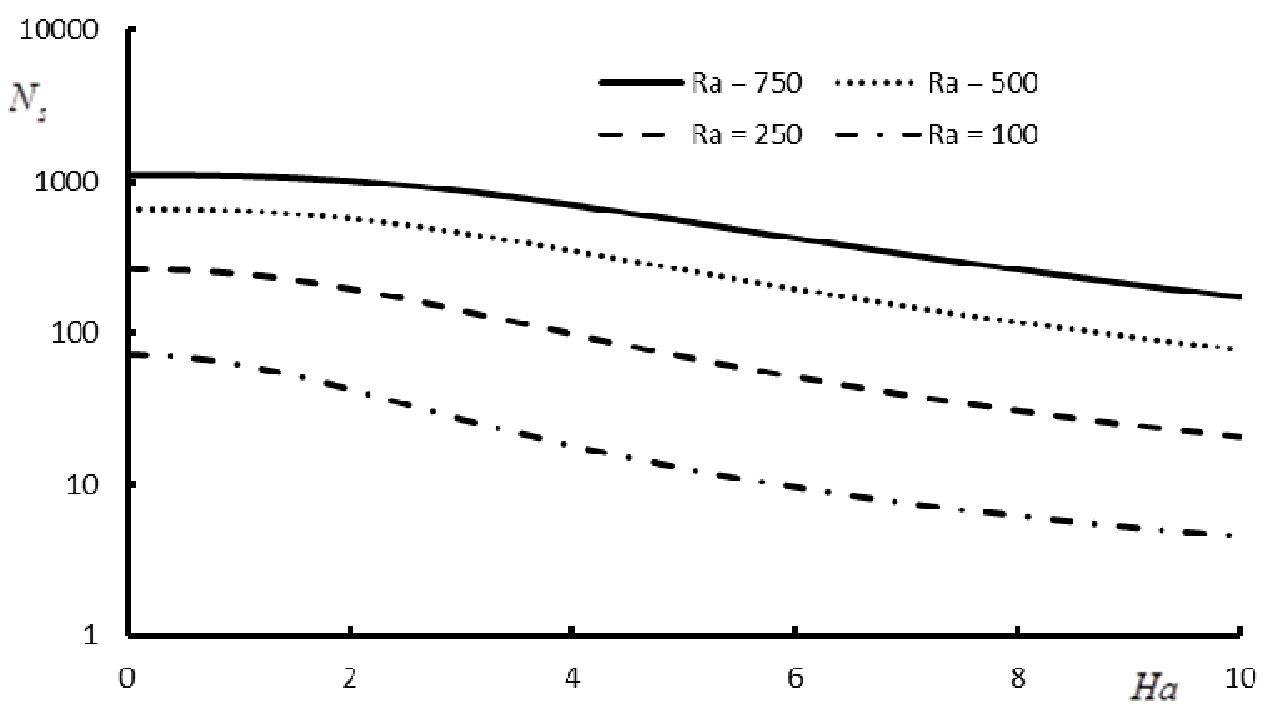

(b)

Figure 4. Variation of $\overline{N u}$ and $N_{s}$ with $H a$ at constant $N_{\mu}=0.1, N=1$ and $L e=1$.

carried out by convection and diffusion and the increase $f$ $\overline{S h}$ is due to the magnifying the convection term (Equation 10).

On the other hand, the heat transfer process is not affected much by increasing the Lewis number. This is due to the fact that there is no clear effect of the concentration variation on the buoyancy forces or the circulation of the fluid in the cavity at low values of $L e$ and $R a$. The strength of the circulation of the fluid in the cavity can be measured by maximum absolute stream function in the cavity. This value is found to be $|\Psi|_{\max }=9.1069$ for the case with $L e=0.1$ and the following parameters: $N_{\mu}$ $=0.1, N=1, H a=0$ and $R a=100$. This leads to a fixed rate of entropy generation, as shown in Figure 5(c), since the mass transfer effect on the entropy generation due to the irreversible generated by viscosity effects. The contour plots for isotherms and iso-concentrations shown 


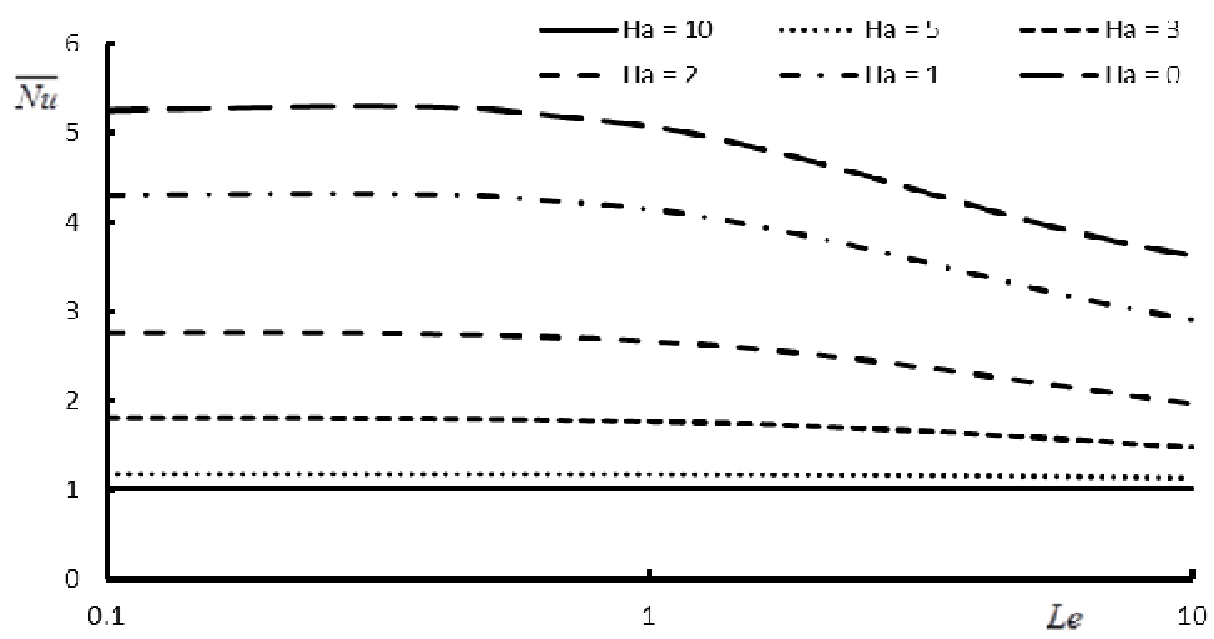

(a)

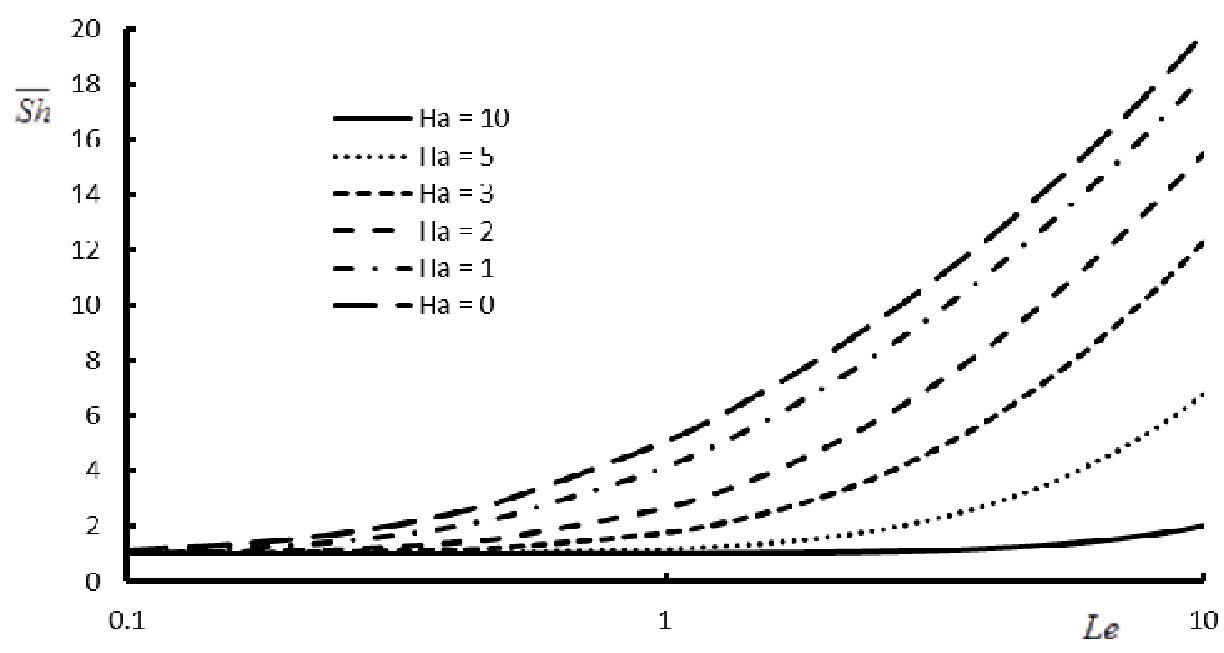

(b)

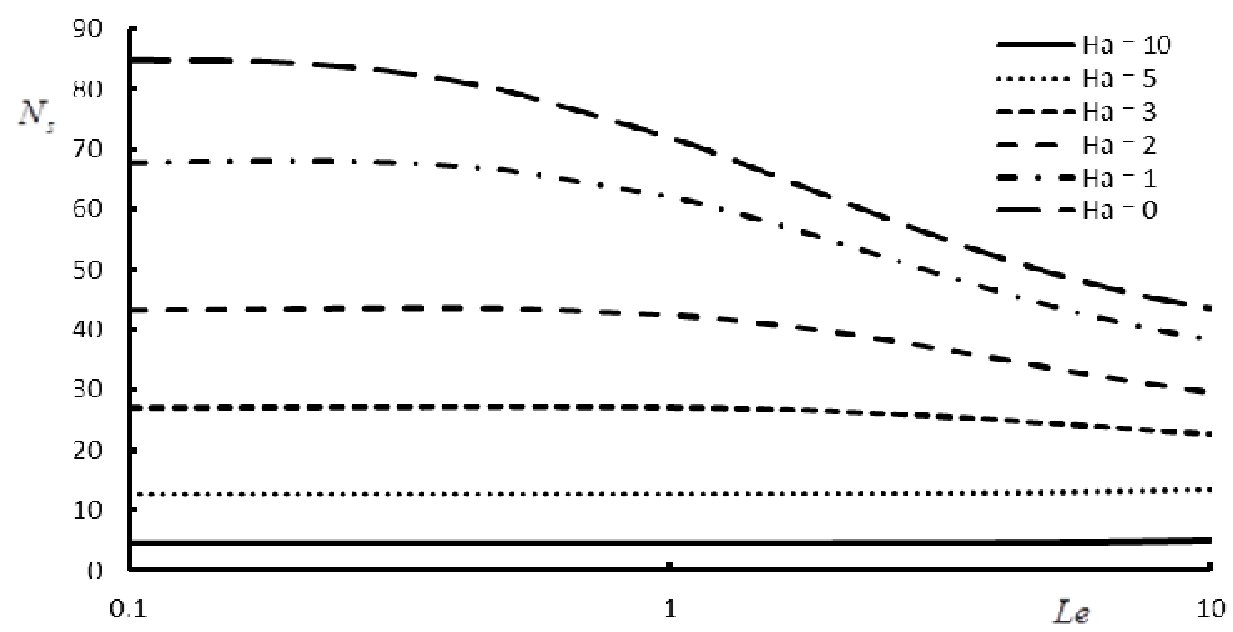

(c)

Figure 5. Variation of $\overline{N u}, \overline{S h}$ and $N_{s}$ with $L e$ at constant $N_{\mu}=0.1, N=1$ and $R a=100$. 
in Figure 6(a) indicate that the mass transfer process is almost pure diffusion at $L e=0.1$ as the contour lines are almost vertical lines. Increasing the Lewis number enhances the convection mode in the mass transfer process. This became obvious by comparing the contour plots for isotherms and iso-concentrations presented in Figures 6(a), (b) and (c) for $L e=0.1, L e=5$ and $L e=10$ respectively. Note that the contour plots for isotherms and iso-concentrations are the same for the case of $L e=$ 1 and almost similar to the isotherms shown in Figure 6 and therefore not shown.

It was observed that the strength of the fluid circulation in the cavity was reduced by increasing the Lewis number. The strengths of the fluid circulation are represented respectively by $|\Psi|_{\max }=9.1069,|\Psi|_{\max }=$ 5.1475 and $|\Psi|_{\max }=4.7883$ for the cases of $L e=0.1$, Le $=5$ and $L e=10$ with fixed following parameters: $N_{\mu}=$ $0.1, N=1, H a=0$ and $R a=100$. Figure 6 shows that the isotherms are not affected much with increasing $L e$ and other fixed parameters compare to the contours of the iso-concentrations. This leads to increase in the average Sherwood number with the increase of $L e$ as shown in Figure 5(b) while the average Nusselt number and the entropy generation are decreasing slightly by increasing Le as shown in Figures 5(a) and (c). Figure 5 shows also that the heat, mass and entropy generation are reduced by increasing the magnetic field and, at $\mathrm{Ha}=10$, the variations of $\overline{\mathrm{Nu}}, \overline{\mathrm{Sh}}$ and $N_{s}$ with $L e$ are almost constant.

\section{Conclusion}

The entropy generation for the combined natural convection heat and mass transfer in a porous cavity subjected to a magnetic field is considered for investigation in the present study. In order to reduce the rate of entropy generation, it is necessary to reduce the reference temperature and reduce the viscosity and the thermal diffusivity of the working fluid. The rate of entropy generation can be minimized for the flow through a high permeable porous medium. The numerical results for $L e$ $=1, H a=0$ and $N=-1$, show a stagnate fluid everywhere in the cavity since the buoyancy forces generated due to temperature and concentration differences are in the same order and opposite directions. In this case, the heat and mass transfer is a pure diffusion process, in which the values of $\overline{N u}$ and $N_{s}$ are the minimum. Increasing or decreasing the value of the buoyancy ratio parameter $(M)$ leads to enhance the fluid circulation due to the increase of the resultant buoyancy force in the cavity. This leads to the increase the values of $\overline{N u}$ and $N_{s}$. The average Sherwood number is increasing with the increase of Lewis number due to the magnifying the convection mode in the mass transfer process. It is observed that the strength of the fluid circulation in the cavity is reduced by increasing the Lewis number. This leads to the decrease slightly in the average Nusselt number and the entropy generation are decreasing by increasing Le. The numerical results show that increasing the magnetic field parameter (Hartmann number) leads to reduce the flow circulation strength in the cavity and this leads to decrease the rate of heat and mass transfer as well as the rate of entropy generation.

\section{Nomenclature}

$\overrightarrow{\mathbf{B}} \quad$ External magnetic field vector, $\left(\mathrm{Wbm}^{-2}\right.$ or Tesla)

C Molar concentration, $\left(\mathrm{mol} . \mathrm{m}^{-3}\right)$

$D$ Effective mass diffusivity through the fluid saturated porous matrix. $\left(\mathrm{m}^{2} \mathrm{~s}^{-1}\right)$

$\overrightarrow{\mathbf{g}} \quad$ Acceleration vector, $\left(\mathrm{ms}^{-2}\right)$

$\mathrm{Ha}$ Hartmann number, Equation (11).

$\overrightarrow{\mathbf{I}} \quad$ Electric current vector, (A)

$K$ Permeability of the porous medium $\left(\mathrm{m}^{2}\right)$

$L \quad$ Cavity length $(m)$

Le Lewis number, Equation (11).

$N \quad$ Buoyancy ratio parameter, Equation (11)

$N_{\mu}$ Irreversibility distribution ratio related to velocity gradients, Equation (16)

$N_{S} \quad$ Dimensionless entropy generation, Equation (17)

$\overline{N u} \quad$ Average Nusselt number, Equation (13)

$p$ pressure, $\left(\mathrm{Nm}^{-2}\right)$

$R \quad$ Universal gas constant $\left(\mathrm{Jkg}^{-1} \mathrm{~K}^{-1}\right)$

Ra Rayleigh number, Equation (11)

$S$ Dimensionless molar concentration, Equation (7)

$\dot{S}_{\text {gen }} \quad$ Entropy generation rate per unit volume $\left(\mathrm{Wm}^{-3} \mathrm{~K}^{-1}\right)$

$\dot{S}_{G E N}$ Dimensionless entropy generation rate per unit volume.

$\overline{S h}$ Average Sherwood numbers, Equation (13)

$T$ fluid temperature $(\mathrm{K})$

$u, v \quad$ Velocity components in $\mathrm{x}, \mathrm{y}$ directions $(\mathrm{m} / \mathrm{s})$

$U, V$ Dimensionless velocity components in $X, Y$ directions, Equation (7)

$\overrightarrow{\mathbf{V}} \quad$ Velocity vector, $\left(\mathrm{ms}^{-1}\right)$

$x, y \quad$ Cartesian coordinates $(\mathrm{m})$

$X, Y$ Dimensionless coordinates, Equation (7)

\section{Greek symbols}

$\alpha \quad$ Effective thermal diffusivity through the fluid saturated porous matrix. $\left(\mathrm{m}^{2} \mathrm{~s}^{-1}\right)$

$\beta_{T} \quad$ Thermal expansion coefficients $\left(\mathrm{K}^{-1}\right)$

$\beta_{c} \quad$ Solute expansion coefficient $\left(\mathrm{m}^{3} \mathrm{~mol}^{-1}\right)$ 

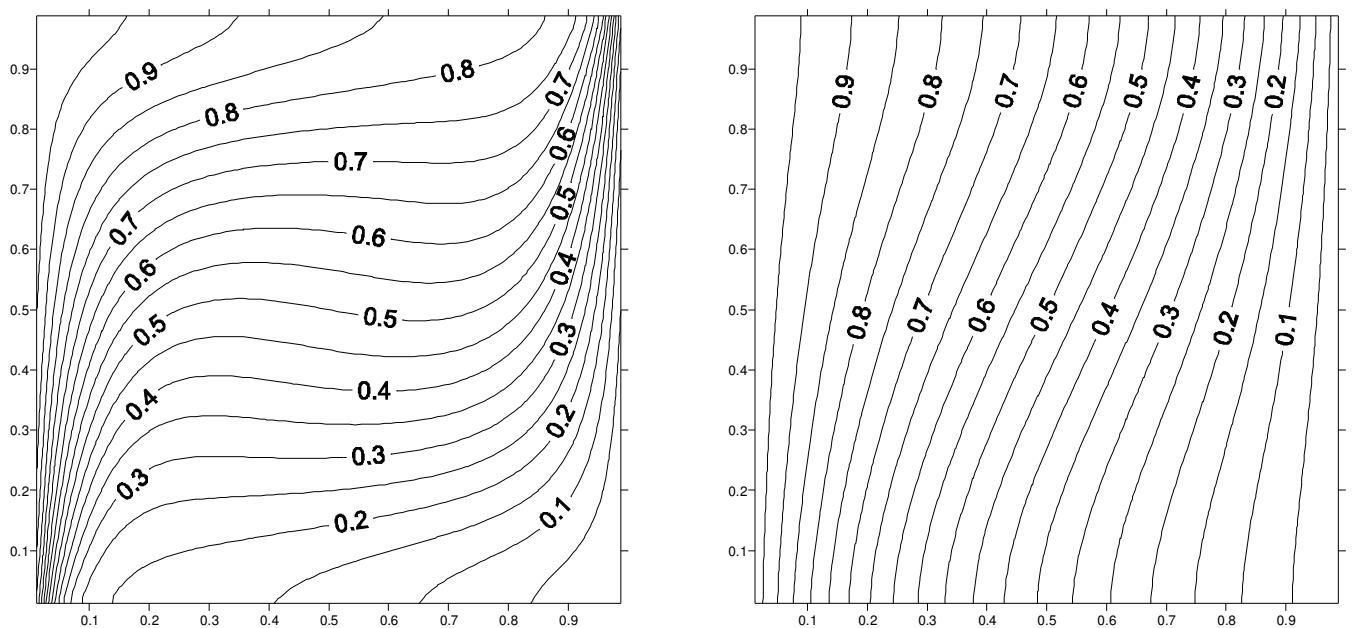

(a)
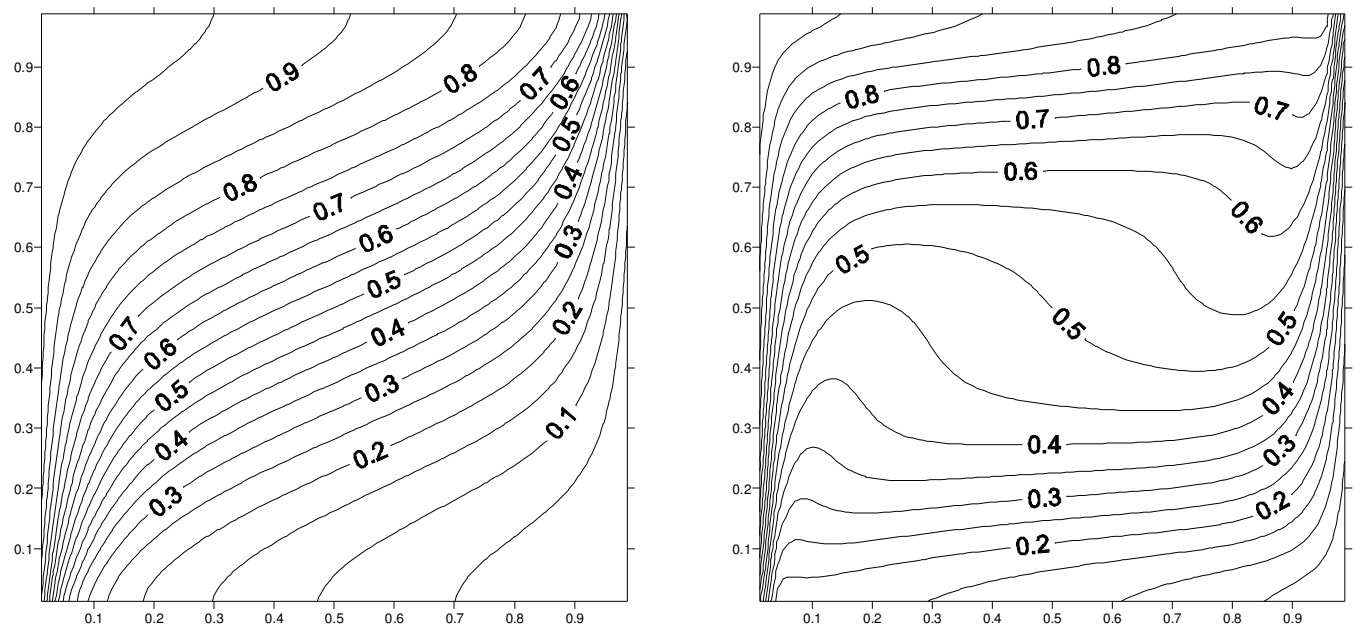

(b)
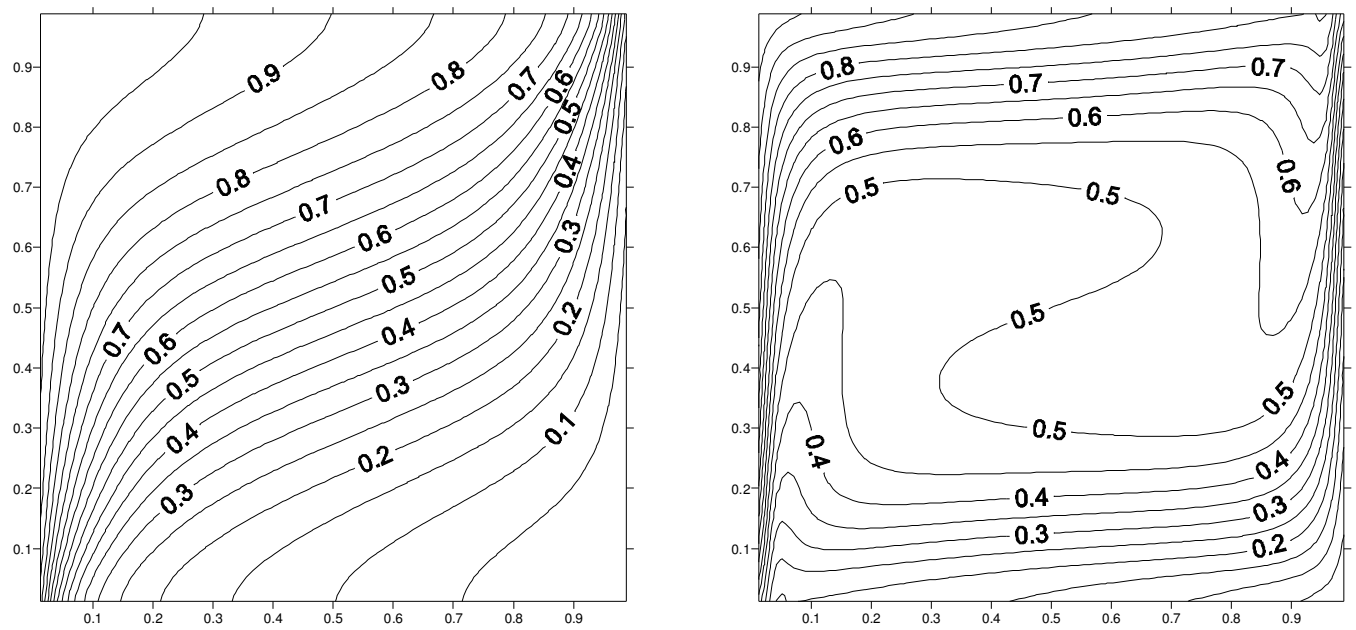

(c)

Figure 6. Isotherms (left) Iso-concentrations (right) with $N_{\mu}=0.1, N=1, H a=0$ and $R a=100$. (a) $L e$ $=0.1\left(|\Psi|_{\max }=9.1069\right)$. (b) $L e=5\left(|\Psi|_{\max }=5.1475\right)$. (c) $L e=10\left(|\Psi|_{\max }=4.7883\right)$. 
$\theta \quad$ Dimensionless temperature, Equation (7)

$\Psi \quad$ Dimensionless stream function.

$\mu \quad$ Dynamic viscosity, $\left(\mathrm{kg} \cdot \mathrm{m}^{-1} \cdot \mathrm{s}^{-1}\right)$

$\rho$ Density, $\left(\mathrm{kg} \cdot \mathrm{m}^{-3}\right)$

$\sigma \quad$ Fluid electrical conductivity, $\left(\Omega^{-1} \mathrm{~m}^{-1}\right)$

$\varphi \quad$ Electric potential, (V).

\section{REFERENCES}

Alchaar S, Vasseur P, Bilgen E (1995). The effect of a magnetic field on natural convection in a shallow cavity heated from below. Chem. Eng. Comm. 134:195-209.

Baytas AC (2000). Entropy generation for natural convection in an inclined porous cavity. Int. J. Heat Mass Transf. 43:2089-2099.

Bian W, Vasseur P, Bilgen E, Meng F (1996). Effect of an electromagnetic field on natural convection in an inclined porous layer. Int. J. Heat Fluid Flow 17:36-44.

Famouri M, Hooman K (2008). Entropy generation for natural convection by heated partitions in a cavity. Int. Comm. Heat Mass Transf. 35:492-502.

Garandet JP, Alboussiere T, Moreau R (1992). Buoyancy driven convection in a rectangular enclosure with a transverse magnetic field. Int. J. Heat Mass Transf. 35:741-748.

Hooman K, Gurgenci H, Merrikh AA (2007). Heat transfer and entropy generation optimization of forced convection in porous-saturated ducts of rectangular cross-section. Int. J. Heat Mass Transf. 50:20512059.

Hooman K, Hooman F, Mohebpour SR (2008). Entropy generation for forced convection in a porous channel with isoflux or isothermal walls. Int. J. Exergy 5:78-96.

Kaluri RS Basak T (2011). Role of entropy generation on thermal management during natural convection in porous square cavities with distributed heat sources. Chem. Eng. Sci. 66:2124-2140.

Kaviany M (1999). Principles of Heat Transfer in Porous Media. Springer, New York. http://books.google.com.ng/books/about/Principles_of_Heat_Transfer in_Porous_Me.html?id=pX61QAOKKqsC\&redir_esc $=y$.

Mahmud S, Fraser RA (2004). Magnetohydrodynamic free convection and entropy generation in a square porous cavity. Int. J. Heat Mass Transf. 47:3245-3256.

Mchirgui A, Hidouri N, Magherbi M, Brahim AB (2012). Entropy generation in double-diffusive convection in a square porous cavity using Darcy-Brinkman formulation. Transp. Porous Med. 93:223-240.
Mojtabi A, Mojtabi MC (2005). Double-Diffusive Convection in Porous Media; in K. Vafai (Ed.), Handbook of Porous Media. $2^{\text {nd }}$ edition, Taylor \& Francis Group, New York http://www.crcnetbase.com/doi/pdf/10.1201/9780415876384.fmatt.

Nield DA, Bejan A (2006). Convection in Porous Media. $3^{\text {rd }}$ ed. Springer, New York. 3rd ed.

Oreper GM, Szekely J (1983). The effect of an externally imposed magnetic field on buoyancy driven flow in a rectangular cavity. $\mathrm{J}$. Cryst. Growth 64:505-515.

Ozoe H (2005). Magnetic Convection. Imperial College Press. pof.aip.org/phfle6/v19/i8/p087104_s1?view=refs.

Patankar SV (1980). Numerical Heat Transfer and Fluid Flow. McGrawHill, New York. www.ewp.rpi.edu/hartford/ ernesto/F2012/.../Patankar-NHTFF1980.pdf.

Pop I, Ingham DB (2001). Convective Heat Transfer: Mathematical and Computational Modelling of Viscous Fluids and Porous Media. Pergamon, Oxford.

Rudraiah N, Venkatachalappa M, Subbaraya CK (1995). Combined surface tension and buoyancy-driven convection in a rectangular open cavity in the presence of a magnetic field. Int. J. Nonlinear Mech. 30:759-770.

Saeid NH, Mohamad AA (2005). Natural convection in a porous cavity with spatial sidewall temperature variation. Int. J. Num. Meth. Heat Fluid Flow 15:555-566.

Saeid NH, Pop I (2004). Transient free convection in a square cavity filled with a porous medium. Int. J. Heat Mass Transf. 47:1917-1924.

Versteeg K, Malalasekera W (2007). An Introduction to Computational Fluid Dynamics: The Finite Volume Method. Pearson Prentice Hall.

Woods LC (1975). The Thermodynamics of Fluid Systems. Oxford University Press: Oxford, UK. 